Early use of polyphenol-rich Cistus creticus extract containing nasopharyngeal spray is associated with significantly shorter duration of symptoms in mild COVID-19 patients: A retrospective case-

\title{
control study
}

\section{Istvan JANKOVICS ${ }^{1}$, Mariann BORSOS ${ }^{2}$, Sara MIRANI $^{3}$, Béla DÉNES ${ }^{4}$}

${ }^{1}$ Complex Medical Centre Déli klinika, Hungary.

${ }^{2}$ Adware Research Ltd., Hungary.

${ }^{3}$ University of Debrecen, Faculty of Pharmacy, Hungary.

${ }^{4}$ Veterinary Diagnostic Directorate, National Food Chain Safety Office, Budapest, Hungary.

*Corresponding Author: Istvan JANKOVICS, Complex Medical Centre Déli klinika, Hungary.

Received date: 12 September 2021; Accepted date: 28 September 2021; Published date: 30 September 2021

Citation: JANKOVICS I, BORSOS M, MIRANI S, DÉNES B (2021) Early use of polyphenol-rich Cistus creticus extract containing nasopharyngeal spray is associated with significantly shorter duration of symptoms in mild COVID-19 patients: A retrospective case-control study. J Comm Med and Pub Health Rep 2(9): https://doi.org/10.38207/JCMPHR/2021/0208155

Copyright: @ 2021 Istvan JANKOVICS. This is an open-access article distributed under the terms of the Creative Commons Attribution License, which permits unrestricted use, distribution, and reproduction in any medium, provided the original author and source are credited.

\begin{abstract}
The majority of COVID-19 occurs as mild upper respiratory tract infection. The most effective tool is a vaccination to prevent unfavorable outcomes. Polyphenolic compounds, including Cistus species, have been shown to exert in vitro activity against viruses causing upper respiratory symptoms, including SARS-CoV-2. There is no data about the clinical impact of the supportive nasopharyngeal application of Cistus creticus extract (CCE) in mild COVID-19. A single-center retrospective case-control study was performed to evaluate the potential efficacy of CCE in a group of 170 PCR confirmed SARS-CoV-2 infected patients. The use of CCE containing nasopharyngeal spray was associated with fewer symptoms on day 12 .
\end{abstract}

Keywords: SARS-CoV-2, COVID-19, Cistus creticus, polyphenols, quality of life

\section{Introduction}

Since the appearance of SARS-CoV-2 in late 2019, the scientific community has focused heavily on the search for effective treatment strategies of COVID-19. The current approach to treatment is based on the severity and actual phase of the disease with risk stratification. There are differences in the guidelines but as a general principle, antiviral treatment (i.e., remdesivir) is recommended in moderate and severe diseases with additional corticosteroid if supplemental oxygen is needed, furthermore if clinical and biochemical indicators of cytokine release syndrome is present additional immunomodulation such as Tocilizumab or Baricitinib may be required [1-3]

There is a paucity of data regarding the usefulness and efficacy of supportive treatment of mild COVID-19, in spite of the fact that the vast majority of the cases contribute to this form of the disease. Several symptomatic and supplementation adjuvant therapies

\section{Methods}

Study design and setting

A single-center, retrospective, case-control study was performed among consecutive adult outpatients with mild COVID-19 confirmed by polymerase chain reaction (PCR) attended at Complex Medical Centre private clinic, Budapest, Hungary between January 15 and May 31, 2021. Only those patients included, who had completed including medicinal herbs were proposed to have potential benefits in mild COVID-19, such as vitamin D, Zinc, Selene, Quercetin, Oleanolic acid, Theaflavin 3,3'-di-O-gallate, Phyllaemblinol, Rosmarinic acid, etc $[4,5]$. Recently, it has been demonstrated that several polyphenolic compounds exert pleiotropic anti-SARS-CoV- 2 efficacy [6]

Cistus creticus, a native medicinal plant in Europe containing a high concentration of polyphenols were proved to develop anti-influenza activity in mice and a randomized clinical study also confirmed the beneficial effect in the upper respiratory tract infections [7-10]. Currently, little is known about the potential supportive effect of CCE polyphenols in the context of COVID-19 symptomatology, thus we designed this study to evaluate the potential effects of this polyphenol-rich extract.

voluntarily at least 3 symptoms related questionnaires during their ambulatory observation period. The use of online self-reported questionnaires for symptoms and quality of life (QoL) were offered as a routine procedure at the clinic that has been approved by the Institutional Ethical Review Board and has been implemented in accordance with the ethical standards. The retrospective analysis and 
assessments of clinical and laboratory data of selected patients recorded within the observation period were performed in accordance with the relevant acts on medical records of the country.

\section{Cistus creticus extract composition}

Cistus creticus Acrocephalus extra., $100 \mathrm{mg} / \mathrm{ml}$; Echinacea purpureae extra., $240 \mathrm{mg} / \mathrm{ml}$; Salvia officinalis extra., $45 \mathrm{mg} / \mathrm{ml}$; Citrus bioflavonoid complex, $20 \mathrm{mg} / \mathrm{ml}$; Acerola dried ext. $120 \mathrm{mg} / \mathrm{ml}$.

\section{Patient eligibility and COVID-19 diagnosis}

All adult ( $>18$ years of age at the time of examination) outpatients attending the clinic with mild (case definition as per WHO severity score) upper respiratory symptoms were screened for inclusion (1). Detection of SARS-CoV-2 in pharyngeal wash samples was performed by RT-PCR amplification of SARS-CoV-2 N-gene fragments, based on WHO guidelines. All PCR confirmed COVID19 patients were included in the study if the onset of symptoms were within 96 hours. Patients with a moderate or severe form of COVID19 (according to the WHO score) or patients referred from inpatient COVID-19 centers were not included.

\section{Data collection, symptom survey, and outcomes}

Data were collected from the institute's electronic patient management framework program. Collected data were basic demographics, comorbidities, comedication, lifestyle factors. A set of questionnaires were developed with a modification of 3 different

\section{Results}

During the study period, there were 170 PCR confirmed SARS-CoV2 patients consecutively included in the study, 86 in the CCE group and 84 in the control group. Baseline demographic and clinical characteristics are shown in Table 1. Mean age was $43.8( \pm 12.8)$ years and were mostly females, significantly more in the CCE group (76.7 \% vs $58.3 \%, \mathrm{p}=0.01$ ). Comorbidities including chronic cardiovascular disease $(12.9 \%)$ were rare and balanced in the groups. The typical patient of the cohort is a young female with no comorbidities. Baseline clinical characteristics and symptomatic treatment other than CCE were balanced except for significantly more patients with fever and fatigue in the CCE group (61.6\% vs $25 \%$, p $<0.001$, and $79.1 \%$ vs $56 \%, \mathrm{p}<0.001$, respectively). As for the 4 validated and standardized tests combined to assess symptoms. First, part of the Wisconsin Upper Respiratory Symptom Survey (WURSS24) was used, which is a validated questionnaire designed to assess the quality of life and symptoms of acute viral respiratory tract infections. Second, the upper respiratory tract infection score, and third, the WHO severity and interference score was also used (1). Patients were asked to complete the test elements battery every second day (day 2, 4, 6, 8, 10 and final evaluation of day 12). The primary outcome was a change in the symptoms on day 12 . The secondary endpoint was SARS-CoV-2 PCR negativity in the nasopharyngeal sample on days 10-15.

\section{Statistical methods}

Continuous variables were described as mean \pm standard deviation (SD), categorical variables were expressed as absolute numbers (n) with percentages $(\%)$. Baseline characteristics were compared by using the Chi-square test and Fisher's exact test for binary variables and t-test for age and time from onset of symptoms. The frequency of symptoms over time was modeled by repeated measures mixed model using PROC GENMOD including day, treatment, and treatment*day as fix factors and using a logit link function. Odds ratios with the $95 \%$ $\mathrm{CI}$ and related $\mathrm{p}$-values are presented for each time point.

For all statistical tests, a 2-tailed $p$-value of $<0.05$ determined statistical significance. Data collection was carried out by the electronic patient management framework program and with Microsoft Office Excel 2016, tests were calculated using SAS 9.4.

co-primary endpoints, patients receiving CCE were less frequently having headache, nasal stuffiness/discharge, sore throat, and nausea ( $\mathrm{p}=0.03, \mathrm{p}=0.01, \mathrm{p}=0.014, \mathrm{p}=0.013$, respectively; on day 12 for headache, nasal stuffiness/discharge, sore throat and on day 10 for nausea; Table 2.). Interestingly, fatigue was more common in the CCE group, and on day 12 was less frequent as detailed in Table 2. The other set of tests and questionnaires have not revealed significant differences in the changes. The time from symptom onset to medical care (also adding CCE in the CCE group) was 3 days. Evaluating SARS-CoV-2 positivity in the nasopharynx, we found that among patients receiving CCE were RNA negative significantly more frequent ( $81 \%$ vs $93 \%, \mathrm{p}=0.019$, Figure 1). 
Table 1. Comparing baseline demographic and clinical characteristics of mild COVID-19 adult patients receiving Cistus creticus extract versus control group

\begin{tabular}{|c|c|c|c|c|}
\hline Baseline characteristics & Total $(n=170)$ & $\begin{array}{l}\text { Cistus creticus } \\
\text { group }(\mathrm{n}=\mathbf{8 6})\end{array}$ & $\begin{array}{l}\text { Control group } \\
(n=84)\end{array}$ & $p$ value \\
\hline Age (years, mean \pm SD) & $43.8( \pm 12.8)$ & $44.2( \pm 14.5)$ & $43.4( \pm 10.9$ & 0.67 \\
\hline Female gender (n, \%) & $115(67.6)$ & $66(76.7)$ & $49(58.3)$ & $\mathbf{0 . 0 1}$ \\
\hline \multicolumn{5}{|l|}{ Comorbidities (n, \%): } \\
\hline - Chronic cardiovascular disease & $22(12.9)$ & $9(10.5)$ & $13(15.5)$ & 0.947 \\
\hline - Contact, inhaled, or ingested allergies & $8(4.7)$ & $4(4.7)$ & $4(4.6)$ & 0.973 \\
\hline - Chronic pulmonary disease & 0 & 0 & 0 & - \\
\hline - Diabetes mellitus & 0 & 0 & 0 & - \\
\hline \multicolumn{5}{|l|}{ Clinical characteristics: } \\
\hline - Fever $(\mathrm{n}, \%)$ & $74(43.5)$ & $53(61.6)$ & $21(25.0)$ & $<0.001$ \\
\hline - Chills (n, \%) & $67(39.4)$ & $33(38.4)$ & $34(40.5)$ & 0.079 \\
\hline - Fatigue (n, \%) & $115(67.6)$ & $68(79.1)$ & $47(56.0)$ & 0.001 \\
\hline - Muscle pain (n, \%) & $80(48.1)$ & $45(52.3)$ & $35(41.7)$ & 0.164 \\
\hline - Sore throat $(\mathrm{n}, \%)$ & $79(46.5)$ & $38(44.2$ & $41(48.8)$ & 0.546 \\
\hline - Cough (n, \%) & $85(50.0)$ & $38(44.2)$ & $47(56.0)$ & 0.125 \\
\hline - Nasal stuffiness/discharge (n, \%) & $68(40.0)$ & $30(34.9)$ & $38(45.2)$ & 0.168 \\
\hline - Headache $(\mathrm{n}, \%)$ & $84(49.4)$ & $43(50.0)$ & $41(48.8)$ & 0.877 \\
\hline - Diarrhoea (n, \%) & $26(15.3)$ & $14(16.3)$ & $12(14.3)$ & 0.718 \\
\hline - Dyspnoe (n, \%) & $25(14.7)$ & $15(17.4)$ & $10(11.9)$ & 0.308 \\
\hline - Wheezing (n, \%) & $8(4.7)$ & $5(5.8)$ & $3(3.6)$ & 0.72 \\
\hline - Chest pain (n, \%) & $22(12.9)$ & $12(14.0)$ & $10(11.9)$ & 0.691 \\
\hline - Abdominal pain (n, \%) & $13(7.6)$ & $6(7.0)$ & $7(8.3)$ & 0.739 \\
\hline - Nausea $(\mathrm{n}, \%)$ & $11(6.5)$ & $7(8.1)$ & $4(4.8)$ & 0.535 \\
\hline $\begin{array}{l}\text { Time from symptom onset to } \\
\text { supportive medical care (including } \\
\text { Cistus creticus } \text { use)(days, mean } \pm \mathrm{SD} \text { ) }\end{array}$ & $3( \pm 0.9)$ & $2.9( \pm 0.9)$ & $3.1( \pm 0.9)$ & 0.189 \\
\hline
\end{tabular}

Table 2. Clinical changes of signs and symptoms in mild COVID-19 adult patients receiving Cistus creticus extract versus control group based on the patient-reported questionnaire during the study period. CCE: Cistus creticus extract group, C: control, OR: odds ratio, CI: $95 \%$ confidence interval

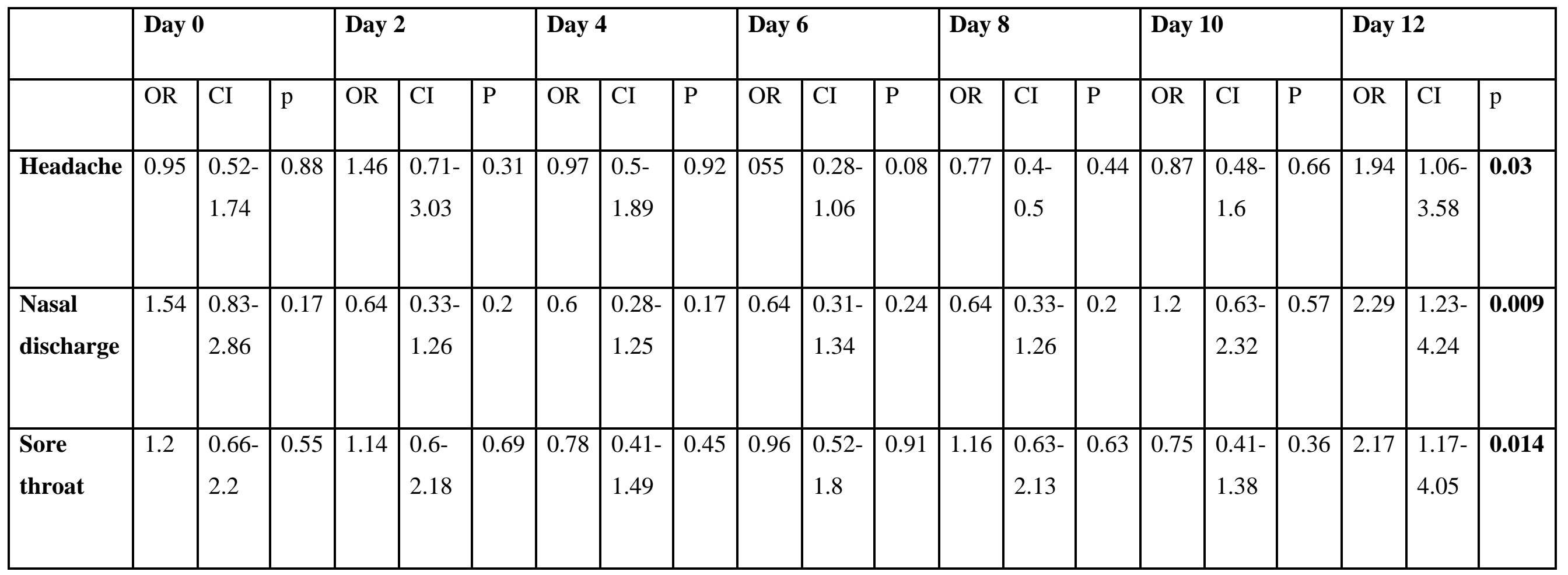




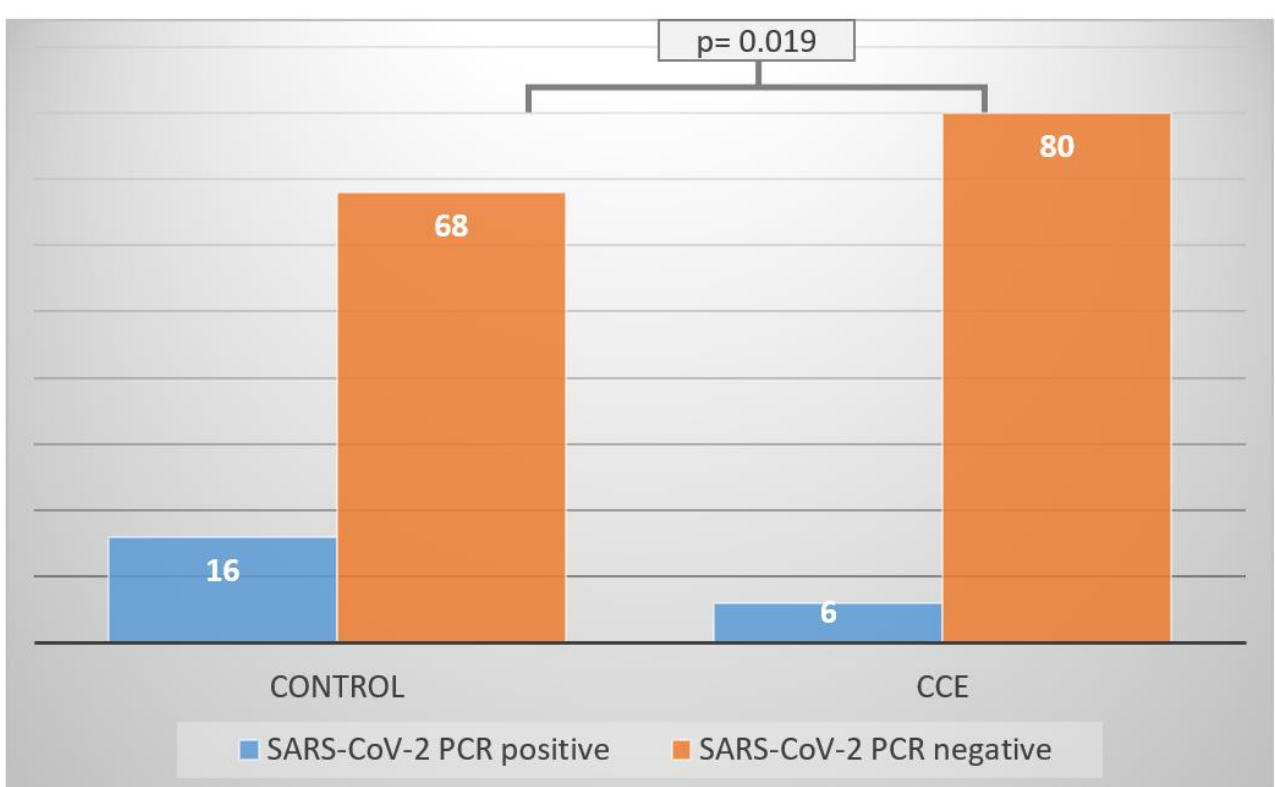

Figure 1. SARS CoV-2 PCR negativity on day 12 of treatment in CCE versus Control group. PCR: polymerase chain reaction, CCE: Cistus creticus extract group

\section{Discussion}

This case-control study of 170 PCR confirmed mild COVID-19 adult patients receiving either Cistus creticus extract added to symptomatic treatment or only symptomatic treatment revealed that on day 12 fewer patients in the CCE group had COVID-19 associated symptoms.

The role of COVID-19 vaccines is of paramount importance in the fight against COVID-19. Preventing critical illness and death caused by COVID-19 is probably the most studied field of medicine in the last two years and should continue until ideal vaccines and medicines are available. However, the vast majority of the COVID-19 cases are asymptomatic or mild presenting as a conventional upper respiratory tract infection well known by all of us. Increasing symptom-free days and improving quality of life in this context is relevant due to the high magnitude affected patients.

It has been shown that Cistus incanus (recently called Cistus creticus) has in vitro effect against influenza A (H7N7) virus in cell culture [7] Further studies confirmed the findings in vivo; treating mice with an aerosol formulation of the extract did not develop diseases and showed no histopathological changes in the bronchioles epithelial cells [8]. Later, Kalus et al performed a retrospective randomized, placebo-controlled trial of 160 patients with upper respiratory tract infection, where the treatment with Cistus creticus extract resulted in a more significant decrease of symptoms based on a subjective scoring system [9]. Interestingly, $46 \%$ of the cases were caused by "virus, other than influenza A and B", suggesting the presence of conventional common cold viruses such as the coronaviruses.

The effect of Cistus creticus extract is associated with its high polyphenolic concentration, which has been shown to exert antiviral activity $[\mathbf{9 , 1 0 ]}$. Recently, several polyphenolic compounds were in vitro and in vivo examined to evaluate their effect on SARS-CoV-2 infectivity $[\mathbf{6}, \mathbf{1 1}]$. These phenols inhibited the viral attachment to the human angiotensin-converting enzyme 2 receptor, thus preventing the entry into the human alveolar epithelial cells.

The clinical impact of these polyphenolic agents is rarely investigated, although their beneficial effect added to the mucosal immunity may decrease viral load. Inoculum effect, as the burden of infective virions, may also play a role in COVID-19 diseases severity [12]. In our study, symptoms showing significant improvement on day 12 were headache, sore throat, nasal discharge, and nausea. This is in line with the above-mentioned observation that polyphenols disrupt receptor binding and foster local/mucosal immunity. Consequently, local polyphenols can provide potential benefits in the early phase of infection. In our study, the time from symptom onset to CCE treatment was 3 days. It is hypothesized, that early administration of polyphenols, as CCE may exert an additional effect to decrease viral burden, potentially the length of symptoms and SARS-CoV-2 RNA positivity rates in nasopharyngeal samples. In summary, the role of potential phytotherapeutic compounds in mild COVID-19 may provide additional benefits [13]

There are several limitations of our study. First, we did not assess laboratory parameters that would reflect additional objective data, except for SARS CoV-2 PCR test on day 0 and day 10-12. Second, patients were self-reporting every second day, however, a decrease in the activity to $80 \%$ was observed. Third, we used the binary method in the course of symptoms to assess changes, which may not reflect optimally the spectrum of symptoms.

Nevertheless, it should be emphasized that this retrospective study took place in Hungary during the third wave of the pandemic. During the third wave, mortality was thirty times higher than during the first wave. In addition, the mortality rate was 25 and 10 percent higher in the 40-49 and 50-59 age groups, respectively, compared to the second wave. The epidemiological PCR examinations showed that several variants of the virus (Alpha B 1.1.7.; Delta B.1617; and Gamma P.1) were detectable in a high percentage of the data of the National Public Health Center during the study period.

An interesting a significant result was found for one of the gastrointestinal symptoms, namely nausea, on day 10 of the study. Given that more and more data currently indicate the importance of multiplication of the virus in the gastrointestinal tract in addition to 
respiratory replication [14], this data may be important in the prevention of infection, following an appropriate large-scale survey. Therefore, in the light of epidemiological data we believe that this case-control study of 170 patients evaluating the effect of adding

\section{Declarations}

Conflict of interest: The authors declare no conflicts of interest regarding this article.

\section{References}

1. https://www.who.int/publications/i/item/WHO-2019-nCoVtherapeutics-2021.2Accessed September 01, 2021

2. COVID-19 Treatment Guidelines Panel. Coronavirus Disease 2019 (COVID-19) Treatment Guidelines. National Institutes of Health.

3. https://www.bhiva.org/Coronavirus-COVID-19Accessed September 01, 2021

4. García R, Hussain A, Koduru P, Atis M, Wilson K, et al. (2021) Identification of potential antiviral compounds against SARSCoV-2 structural and non-structural protein targets: A pharmacoinformatic study of the CAS COVID-19 dataset. Comput Biol Med. 133: 104364.

5. Patel B, Sharma S, Nair N, Majeed J, Goyal RK, et al. (2021) Therapeutic opportunities of edible antiviral plants for COVID19. Mol Cell Biochem. 476(6): 2345-2364.

6. Goc A, Sumera W, Rath M, Niedzwiecki A (2021) Phenolic compounds disrupt spike-mediated receptor-binding and entry of SARS-CoV-2 pseudo-virions. PLoS One. 16(6): e0253489.

7. Ehrhardt C, Hrincius ER, Korte V, Mazur I, Droebner K, et al. (2007) A polyphenol rich plant extract, CYSTUS052, exerts anti influenza virus activity in cell culture without toxic side effects or the tendency to induce viral resistance. Antiviral Res. 76(1): 3847.

8. Droebner K, Ehrhardt C, Poetter A, Ludwig S, Planz O (2007) CYSTUS052, a polyphenol-rich plant extract, exerts antiinfluenza virus activity in mice. Antiviral Res. 76(1): 1-10. polyphenolic rich Cistus creticus extract to supportive treatment in mild COVID-19 adult patients showed its efficacy by reducing the length of symptoms.

Funding: Not applicable

Acknowledgements: None declared

9. Kalus U, Grigorov A, Kadecki O, Jansen JP, Kiesewetter H, et al. (2009) Cistus incanus (CYSTUS052) for treating patients with infection of the upper respiratory tract. A prospective, randomised, placebo-controlled clinical study. Antiviral Res. 84(3): 267-71.

10. Kalus U, Kiesewetter H, Radtke H (2010) Effect of CYSTUS052 and green tea on subjective symptoms in patients with infection of the upper respiratory tract. Phytother Res. 24(1): 96-100.

11. Di Pierro F, Derosa G, Maffioli P, Bertuccioli A, Togni S, et al. (2021) Possible Therapeutic Effects of Adjuvant Quercetin Supplementation Against Early-Stage COVID-19 Infection: A Prospective, Randomized, Controlled, and Open-Label Study. Int J Gen Med. 14: 2359-2366.

12. Guallar MP, Meiriño R, Donat-Vargas C, Corral O, Jouvé N, et al. (2020) Inoculum at the time of SARS-CoV-2 exposure and risk of disease severity. Int J Infect Dis. 97: 290-292.

13. Seifert G, Jeitler M, Stange R, Michalsen A, Cramer H, et al. (2020) The Relevance of Complementary and Integrative Medicine in the COVID-19 Pandemic: A Qualitative Review of the Literature. Front Med (Lausanne). 7: 587749.

14. Ding S, Liang TJ (2020) Is SARS-CoV-2 Also an Enteric Pathogen with Potential Fecal-Oral Transmission? A COVID-19 Virological and Clinical Review. Gastroenterology. 159(1): 5361. 